\title{
Monolithic solar water splitting: introducing porosity in multijunction solar cells with minimal degradation to enable ionic shortcuts
}

\author{
Christos Trompoukis ${ }^{1,2, *}$, Tom Bosserez ${ }^{2}$, Aimi Abass ${ }^{3}$, Jan-Willem Schüttauf ${ }^{4}$, Jan Ronge ${ }^{2}$, Johan Martens ${ }^{2}$, \\ Roel Baets ${ }^{1}$ \\ ${ }^{1}$ Ghent University, Photonics Research Group, iGent, Technologiepark Zwijnaarde 15, Ghent, Belgium \\ ${ }^{2}$ KU Leuven, Center for Surface Chemistry and Catalysis, Celestijnenlaan 200F, Leuven, Belgium \\ ${ }^{3}$ Institute of Nanotechnology, Karlsruhe Institute of Technology (KIT), P.O. Box 3640, 76021 Karlsruhe, Germany \\ ${ }^{4}$ CSEM, Rue Jaquet-Droz 1, Neuchâtel, Switzerland \\ christos.trompoukis@ugent.be
}

\begin{abstract}
We propose a new design for monolithic solar water splitting based on porous multijunction solar cells. Porosity, causing minimal solar cell degradation, minimizes the ohmic losses associated to ion transport, maintaining high efficiencies when up-scaling.

OCIS codes: (350.6050) Solar energy; (130.5990) Semiconductors; (310.6845) Thin film devices and applications
\end{abstract}

\section{Introduction}

Energy storage in chemical bonds, e.g. hydrogen, could tackle the problem of the intermittency of renewable energy sources and enable zero carbon emission 100\% renewable energy. Although promising concepts for solar hydrogen production have already been demonstrated (e.g. solar electrolysis, thermal conversion and molecular artificial photosynthesis among others [1,2]), none of the current systems fulfills all four requirements for practical implementation: (i) high solar to hydrogen efficiency (STHE), (ii) low cost, (iii) durability and (iv) scalability. Compared to the already available hydrogen producing systems, based on coupling photovoltaics to electrolysis units, exhibiting high STHE (around 8\% for industrial systems [3], 14.2\% for experimental concepts [4] and 24.4\% for systems with solar concentrators [5]), the photoelectrochemical (PEC) approach offers the possibility for high STHE at low cost. Such PEC devices typically consist of semiconductor photoabsorbers directly interfaced with electrocatalyst layers, a design with various benefits. Firstly, a PEC device can be fabricated as a single monolith where hydrogen and oxygen are produced at separate sides thus avoiding safety concerns while excluding the need for post-production gas separation. Secondly, PEC devices can be made entirely from inorganic materials offering chemical robustness, durability, cost-effectiveness and large-scale fabrication. Finally, monolithic PEC cells require fewer packaging components and peripherals making them cost-efficient.

So far, a PEC device reaching an STHE as high as $12.4 \%$ has been demonstrated [6]. However, in this work expensive III-V materials were used which are too costly for terrestrial applications. In a cost-effective approach based on a triple junction amorphous silicon $(\mathrm{aSi})$ solar cell a $4.7 \%$ device has been reported [7] while recently a device reaching an STHE of $9.5 \%$ was fabricated [8].

As shown in [7], the STHE decreases from $4.5 \%$ to $2.5 \%$ when a wireless configuration is used, due to long ionic transport distances resulting in high ohmic losses which are a major bottleneck for reaching high STHE values. In order to go beyond the state-of-the-art we propose the introduction of pores on monolithic PEC cells as ionic shortcuts so as to maintain high STHE values when up-scaling. We focus on triple junction thin-film silicon solar cells based on amorphous silicon $(\mathrm{aSi})$ and microcrystalline silicon $(\mu \mathrm{cSi})$ as the photoactive layers since this PV technology is capable of providing high as well as easily tunable voltage and current density values with the additional benefit of offering photoactive materials that are expected to be less vulnerable to damage from the pore fabrication process.

\section{Simulation results}

To investigate the potential of the porous monoliths, preliminary 2D electrochemical simulations have been done using Comsol. The system is periodic in the horizontal direction as shown in Fig. 1(a), the reaction kinetic follow the Butler-Volmer equation while platinum and ruthenium oxide are used as the catalysts. Instead of tackling the whole photovoltaic-electrochemical system simultaneously, we calculate the current density-voltage (JV) behavior of each system separately and consider the operating point to be the crossover when the photovoltaic JV curve intersects with the electrochemical JV curve. 

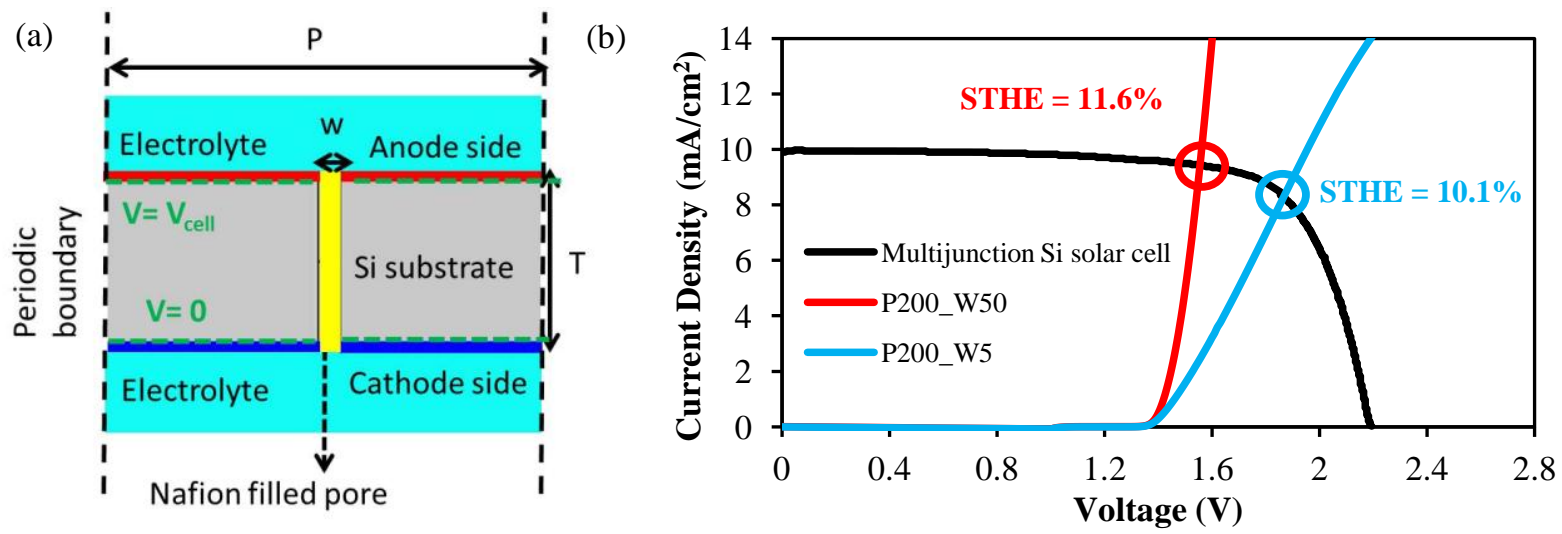

Figure 1. (a) 2D simulated PEC system and (b) realistic achievable STHE of porous PV monoliths.

The JV characteristics for two porous monolithic geometries are shown in Fig. 1(b). Here, we consider that the electrolyte has a $\mathrm{pH}=2$ and the monolith has a thickness of $10 \mu \mathrm{m}$ (a typical thickness of multijunction thin-film solar cells). Calculation results for two topography cases are shown, i.e. a period of $200 \mu \mathrm{m}$ and a pore diameter of $50 \mu \mathrm{m}$ (red curve) and a period of $200 \mu \mathrm{m}$ and a pore diameter of $5 \mu \mathrm{m}$ (blue curve). In the latter case, the ions would have to travel a significantly larger distance and thus suffer from more effective electrolyte resistance. In order to show the potential of the porosity concept and what could be some realistic achievable STHEs we considered the best reported triple solar cell [9]. As highlighted in Fig. 1(b), an STHE of 11.66\% is possible (red circle on Fig. 1(b) highlighting the operating point), assuming no degradation of the PV during pore fabrication. The STHE decreases down to $10.1 \%$ (blue circle on Fig. 1(b) highlighting the operating point) when a less dense pore topography is present, highlighting the impact of ion transport on the device performance.

\section{Experimental results}

In order to investigate to what extent porosity affects the solar cell operation we focused on real aSi and $\mu \mathrm{cSi}$ single junction as well as multijunction ( $\mu \mathrm{cSi}$ bottom cell/aSi middle cell/aSi top cell) solar cells. All solar cells were fabricated by CSEM as reported elsewhere [10], a square array of cylindrical holes with a period of $200 \mu \mathrm{m}$ and a pore diameter of $20 \mu \mathrm{m}$ were fabricated by optical lithography and dry etching while the solar cells were characterized by JV measurements under 1-sun using a solar simulator from Oriel. Plotting the absolute difference (initial value minus value after the implementation of pores in the device, in arbitrary units) of the solar cell parameters, i.e. short circuit current $\left(\mathrm{J}_{\mathrm{sc}}\right.$ in $\left.\mathrm{mA} / \mathrm{cm}^{2}\right)$, open circuit voltage $\left(\mathrm{V}_{\mathrm{oc}}\right.$ in $\left.\mathrm{mV}\right)$, fill factor $(\mathrm{FF}$ in $\%)$ and efficiency ( $\eta$ in \%), before and after introducing porosity, we can notice some trends as shown in Fig. 2(a).

(a)

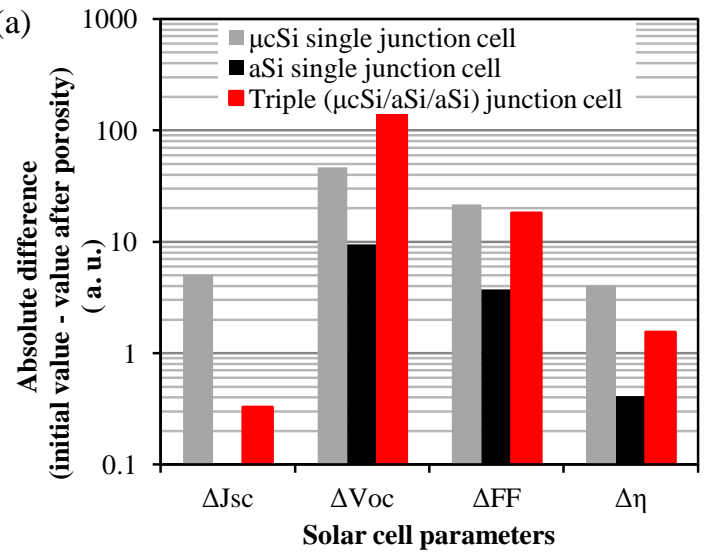

(b)

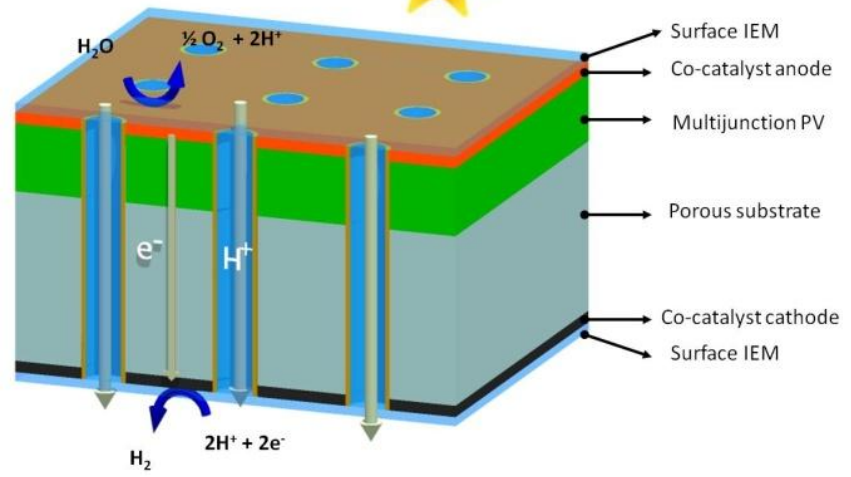

Figure 2. (a) Impact of porosity on solar cell parameters (e.g. $\Delta J_{s c}=$ initial $J_{s c}-J_{s c}$ of porous device) where $\Delta J s c$ is given in units of $\mathrm{mA} / \mathrm{cm}^{2}$, $\triangle V o c$ in units of $m V, \triangle F F$ and $\triangle \eta$ in units of $\%$ and (b) proposed porous monolithic PEC device

First of all, the impact of porosity is more pronounced on the $\mu \mathrm{cSi}$ than the aSi solar cells. On one hand, this happens due to the incomplete light absorption, especially for long wavelength photons, of the indirect band-gap $\mu \mathrm{cSi}$ which porosity further enhances. While several $\mathrm{mA} / \mathrm{cm}^{2}$ were lost when introducing porosity on the $\mu \mathrm{cSi}$ solar cells, the loss was restricted to below $1 \mathrm{~mA} / \mathrm{cm}^{2}$ for the porous aSi solar cells. On the other hand, the defects 
introduced on the photoactive material by the implementation of porosity resulted in $\sim 45 \mathrm{mV}$ of decrease for $\mu \mathrm{cSi}$ solar cells while the effect of porosity on aSi is found to be minimal $(<10 \mathrm{mV})$. The lower diffusion lengths for the aSi solar cells compared to the $\mu \mathrm{cSi}$ solar cells are responsible for such a difference $\mathrm{V}_{\text {oc }}$ as less generated carriers reach the pore sidewalls which act as recombination sites. A similar behavior is seen for the FF upon the introduction pores, although this can be partially attributed to the impact of porosity on the $\mathrm{ZnO}$ contacts, i.e. higher sheet resistance values (results not shown here). Finally, while the impact of porosity on the efficiency of $\mu \mathrm{cSi}$ solar cells was high (loss of $4 \%$ ), aSi solar cells suffer only a limited impact (loss of $0.4 \%$ ). For the multijunction case ( $\mu \mathrm{cSi}$ bottom cell/aSi middle cell/aSi top cell), the triple junction solar cell had only a marginal decrease in $\mathrm{V}_{\text {oc }}$ around $150 \mathrm{mV}$ (from an initial value of $2230 \mathrm{mV}$ to $2080 \mathrm{mV}$ after the implementation of the pores), highlighting the minimal device degradation when implementing pores in the device. Such a high $\mathrm{V}_{\text {oc }}$ achieved for the porous multijunction solar cells could enable high STHE efficiencies and maintain them when upscaling the monolithic device.

From this work and our analysis of the impact of porosity above, we will present our envisioned porous monolithic solar water splitting device, shown in Fig. 2(b). The porous carrier substrate is a highly doped silicon wafer on which porosity is implemented by means of deep reactive ion etching. The triple junction solar cell is based on aSi solar cells only, so as to keep any degradation minimal. In particular, aSi and aSiGe materials with different bandgaps are used. We will discuss design guidelines and experimental considerations in order to enable an STHE of around $8.5 \%$ and further improvements on the design and the solar cells which could lead to higher efficiencies. The impact of implementing pores with dimensions relevant for photonic light trapping [11, 12] in the triple junction water spitting device in order to enable a combination of short ionic distances and light absorption enhancement will also be discussed. Finally, results based on our test vehicle structure (platinum nanoparticle decorated porous silicon photoelectrodes $\left(\Delta \mathrm{E}^{0}=475 \mathrm{mV}\right)$ immersed in a sulfite scavenger $\left(\Delta \mathrm{E}^{0}=170 \mathrm{mV}\right)$ in absence of electrical bias) will be show to demonstrate the beneficial effect of pores on the hydrogen production.

\section{Acknowledgements}

C. Trompoukis and J. Ronge acknowledge the Research Foundation - Flanders (FWO) for a postdoctoral funding. J. Martens and R. Baets are supported by the Flemish government (Methusalem long-term structural funding).

\section{References}

[1] K. Rajeshwar et al., "Solar Hydrogen Generation - Towards a Renewable Energy Future", Springer, New York (2008)

[2] C. A. Grimes et al., "Light, Water, Hydrogen - The Solar Generation of Hydrogen by Water Photoelectrolysis", Springer, New York (2008)

[3] R. Van De Krol and M. Gratzel, "Photoelectrochemical hydrogen production”, Springer, New York (2012)

[4] J-W Schuttauf and M. A. Modestino et al., "Solar-to-hydrogen production at $14.2 \%$ efficiency with silicon photovoltaics and earth-abundant electrocatalysts", J. Electrochem. Soc. 163 (10), F1177-F1181 (2016)

[5] Nakamura et al., "A $24.4 \%$ solar to hydrogen energy conversion efficiency by combining concentrator photovoltaic modules and electrochemical cells”, Appl. Phys. Express 8 (10), 107101 (2015)

[6] O. Khaselev and J. A. Turner, "A Monolithic Photovoltaic-Photoelectrochemical Device for Hydrogen Production via Water Splitting”, Science 280, 425 (1998)

[7] S. Y. Reece et al., "Wireless Solar Water Splitting Using Silicon-Based Semiconductors and Earth-Abundant Catalysts", Science 334, 645648 (2011).

[8] F. Urbain et al., "Multijunction Si photocathodes with tunable photovoltages from $2.0 \mathrm{~V}$ to $2.8 \mathrm{~V}$ for light induced water splitting", Energy Environ. Sci. 9, 145 (2016)

[9] S. Kim et al., "Remarkable progress in thin-film silicon solar cells using high-efficiency triple-junction technology", Sol. Energy Mater. Sol. Cells 119, 26 (2013)

[10] J.-W. Schüttauf et al., "Thin-film silicon triple-junction solar cells on highly transparent front electrodes with stabilized efficiencies up to 12.8\%”, IEEE J. Photovolt. 4, 757-762 (2014)

[11] C. Trompoukis et al., "Photonic nanostructures for advanced light trapping in thin crystalline silicon solar cells", Phys. Status Solidi A 212 $(1), 145-155(2015)$

[12] C. Trompoukis et al., "Disordered nanostructures by hole-mask colloidal llithography for advance light trapping in silicon solar cells", Opt. Express 24 (2), A191-A201 (2016) 